\title{
Trace Transitioning and Exception Handling in a Trace-Based JIT Compiler for Java
}

\author{
CHRISTIAN HÄUBL, Johannes Kepler University Linz \\ CHRISTIAN WIMMER, Oracle Labs \\ HANSPETER MÖSSENBÖCK, Johannes Kepler University Linz
}

Trace-based Just-In-Time (JIT) compilation generates machine code for frequently executed paths (so-called traces) instead of whole methods. While this has several advantages, it complicates invocation of compiled traces as well as exception handling, so that previous trace-based compilers limited the way in which traces could be invoked.

We present a significantly enhanced trace-based compiler where arbitrary transitions between interpreted and compiled traces are possible. For that, we introduce suitable trace calling conventions and extend exception handling to work both within traces and across trace boundaries. Furthermore, we use the recorded trace information for optimizations and combine the tracing ideas with ideas from partial-method compilation to avoid code bloat.

An extensive evaluation with the benchmark suites DaCapo 9.12 Bach and SPECjvm2008 shows that our trace-based compiler achieves up to $59 \%$ higher peak performance than the method-based Java HotSpot client compiler. On a few benchmarks, our fairly simple trace-based compiler shows a higher peak performance than the Java HotSpot server compiler, which is one of today's best optimizing JIT compilers for Java.

Categories and Subject Descriptors: D.3.4 [Programming Languages]: Processors_Compilers, Optimization

General Terms: Algorithms, Languages, Performance

Additional Key Words and Phrases: Java, trace-based, just-in-time, compilation, HotSpot

ACM Reference Format:

Christian Häubl, Christian Wimmer, and Hanspeter Mössenböck. 2014. Trace transitioning and exception handling in a trace-based JIT compiler for java. ACM Trans. Architec. Code Optim. 11, 1, Article 6 (February 2014), 26 pages.

DOI: http://dx.doi.org/10.1145/2579673

\section{INTRODUCTION}

Trace-based JIT compilers compile frequently executed paths, so-called traces that are recorded during execution [Bala et al. 2000]. Figure 1(a) shows the control flow graphs of two methods, while Figure 1(b) shows three traces that span both methods. All traces start at block 1, which is their trace anchor. Which basic blocks are selected as trace anchors depends on the trace recording implementation, as there are multiple ways for detecting trace anchors [Duesterwald and Bala 2000; Hiniker et al. 2005; Bebenita et al. 2010b].

New article, not an extension of a conference paper.

Authors' addresses: Christian Häubl and Hanspeter Mössenböck, Institute for System Software, Johannes Kepler University Linz, Altenbergerstrasse 69, 4040 Linz, Austria; email: haeubl@ssw.jku.at, moessenboeck@ ssw.jku.at; Christian Wimmer, Oracle Labs, USA; email: christian.wimmer@oracle.com.

Permission to make digital or hard copies of part or all of this work for personal or classroom use is granted without fee provided that copies are not made or distributed for profit or commercial advantage and that copies show this notice on the first page or initial screen of a display along with the full citation. Copyrights for components of this work owned by others than ACM must be honored. Abstracting with credit is permitted. To copy otherwise, to republish, to post on servers, to redistribute to lists, or to use any component of this work in other works requires prior specific permission and/or a fee. Permissions may be requested from Publications Dept., ACM, Inc., 2 Penn Plaza, Suite 701, New York, NY 10121-0701 USA, fax +1 (212) 869-0481, or permissions@acm.org.

(c) 2014 ACM 1544-3566/2014/02-ART6 $\$ 15.00$

DOI: http://dx.doi.org/10.1145/2579673 


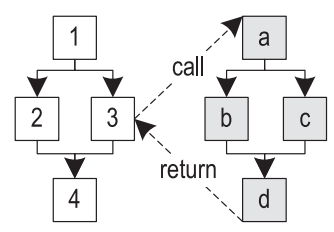

(a) control flow graphs

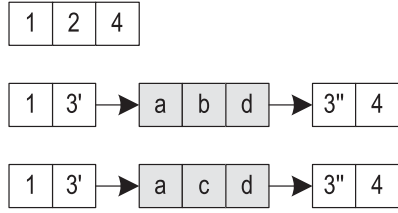

(b) possible traces

Fig. 1. Possible traces through two methods.

A trace consists of a sequence of executed instructions and may also keep track of observed types or values that can be used for aggressive compiler optimizations. If aggressive optimizations are performed based on optimistic assumptions, the compiler emits runtime checks to guard those assumptions. When a runtime check fails, execution falls back to the interpreter. The same fall-back mechanism is also used when a method part must be executed that was not yet compiled. This is necessary because the recorded traces only cover the most frequently executed method parts so that tracebased compilation may result in less generated machine code and faster compilation.

In previous work [Häubl and Mössenböck 2011; Häubl et al. 2012, 2013], we implemented a trace recording infrastructure and a simple trace-based JIT compiler by modifying Oracle's Java HotSpot Virtual Machine (VM). This article contributes the following new aspects:

-We describe a novel and flexible trace transitioning system that allows arbitrary transitions between interpreted and compiled code. We compare our system to other trace-based and method-based JIT compilers.

-We extend our simple trace-based compiler so that it can now also call separately compiled loop traces from compiled code. We discuss issues that arise in this context. To address those issues, and to simplify the invocation of loop traces, we propose to check certain invariants for loop traces before compiling loops separately.

-We extend our trace compiler with a new approach for generalized exception handling in a way that exceptions can be thrown and caught efficiently within traces or across trace boundaries.

-We evaluate our trace-based compiler in terms of peak performance, amount of generated machine code, and compilation time for the benchmark suites SPECjvm2008 [Standard Performance Evaluation Corporation 2008] and DaCapo 9.12 Bach [Blackburn et al. 2006]. The results are compared to the method-based Java HotSpot client and server compilers.

\section{PREVIOUS WORK}

In previous work [Häubl and Mössenböck 2011; Häubl et al. 2012, 2013], we implemented a trace recording infrastructure and a simple trace-based JIT compiler for Java based on the production-quality Java HotSpot VM. Our implementation supports two different kinds of traces: loop traces anchored at loop headers, and method traces anchored at method entries. Figure 2 shows the runtime system of this VM.

Execution of a Java application starts with the class loader that loads, parses, and verifies the class files. This results in runtime data structures such as the constant pool and method objects. Then, a static loop analysis step is performed on the loaded bytecodes to detect loop headers and to create tracing-specific data structures. This explicit loop detection step avoids the detection of false loops [Hayashizaki et al. 2011], which may occur in other approaches where every backwards branch target is assumed 


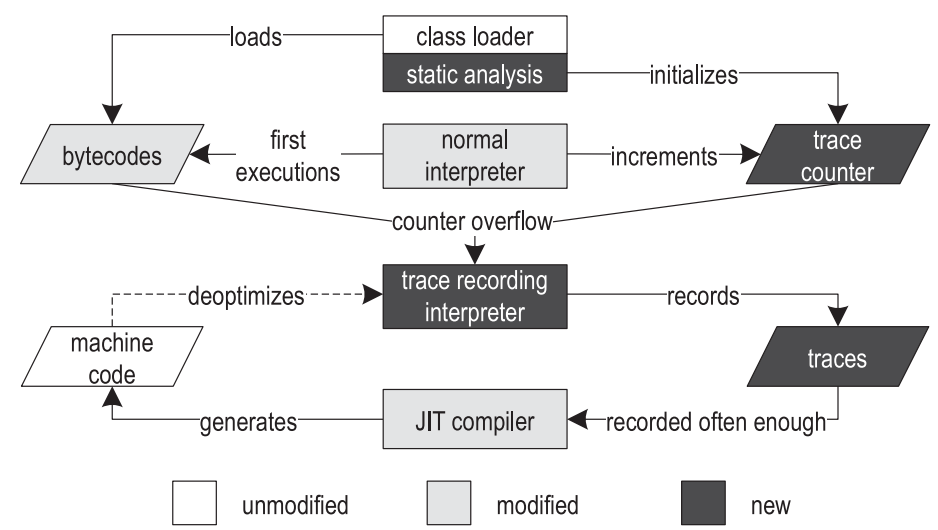

Fig. 2. Trace-based Java HotSpot VM.

to be a potential loop header. The detected loop headers and all method entries are then used as potential trace anchors.

\subsection{Trace Recording}

The Java HotSpot VM uses an interpreter that consists of hand-written assembler templates [Griesemer 1999]. Our trace-based Java HotSpot VM uses two different versions of this template-based interpreter. Execution starts in the normal interpreter, which counts the execution frequencies of trace anchors. This avoids trace recording for rarely executed code parts and allows executing bytecodes at nearly the same speed as the interpreter of the unmodified VM. When a trace anchor is executed frequently enough, it is marked as hot and execution switches to the trace recording interpreter. This interpreter is instrumented to record the executed path and the observed type information in a thread-local buffer. Traces that start at this hot anchor are then recorded for a certain number of times until they are considered hot and therefore compiled to optimized machine code.

Other trace-based compilers typically store information about encountered bytecodes in a single trace, even if the trace crosses method boundaries. So, inlining is done during trace recording [Bala et al. 2000; Bebenita et al. 2010b; Gal and Franz 2006; Gal et al. 2006; Chang et al. 2009; Gal et al. 2009; Inoue et al. 2011; Bolz et al. 2009] and may result in large traces that cannot be split and must be compiled as a whole. We use a different approach. When a method invocation is encountered during trace recording, a separate trace is started for the invoked method and this separate trace is linked to its caller trace [Häubl and Mössenböck 2011]. This restricts individual traces to span at most one method while the linking preserves the call structure and also makes the traces context sensitive: every call site knows exactly which traces were called there. The resulting data structure is similar to the dynamic call graph of the executed application. This allows delaying any inlining decisions to the time of JIT compilation when more information is available.

After a trace has been recorded, our system checks if the trace should be stored or if it was already recorded before. If the trace was already recorded, we just increment a counter in the stored trace to record the execution frequency. Otherwise, the threadlocal trace is copied to globally accessible memory and stored in the list of all traces for that trace anchor.

Our trace recording approach supports all Java features such as exception handling, Java subroutines, reflection, and invocation of native methods. All frequently executed 
trace recording operations, such as recording information for specific bytecodes, are directly implemented in the assembler templates of the trace recording interpreter. More complex operations, such as storing the recorded traces, are implemented in the C-based runtime of the interpreter.

To simplify the detection of already recorded traces, each trace is identified by a hash code. This hash code is computed in a way in which two traces that took the same path but invoked different traces have different hash codes. This ensures that context-sensitive information is preserved over method boundaries. Our trace recording approach would allow recording context-sensitive call information for every executed path through the whole application. However, this would result in a large number of traces for complex applications. To reduce the number of recorded traces to a reasonable amount, loop traces and traces for recursive method invocations are not linked to their parent trace so that the hash code of the parent trace is not modified. So, context sensitivity is preserved over method boundaries but not over loops or recursive methods.

After performing trace recording a certain number of times for a trace anchor, the recorded traces are compiled to optimized machine code. When execution reaches a trace anchor for which compiled machine code already exists, this machine code is invoked instead of interpreting the bytecodes. More details about our trace recording approach can be found in Häubl and Mössenböck [2011].

\subsection{Compilation}

Oracle's Java HotSpot VM ships with two different JIT compilers that share most parts of the VM infrastructure. The client compiler is a simple method-based compiler that is designed for the best startup performance and implements only basic optimizations to achieve a decent peak performance [Kotzmann et al. 2008]. Compilation starts with generating the High-level Intermediate Representation (HIR) that is in Static Single Assignment (SSA) form [Cytron et al. 1991] and represents the control flow graph. On this level, optimizations such as constant folding, null-check elimination, and method inlining are applied. The resulting optimized HIR is used to generate the Low-level Intermediate Representation (LIR), which is close to machine code but still mostly platform independent. The LIR is used for linear scan register allocation [Wimmer and Mössenböck 2005] and translation to machine code.

The server compiler uses partial-method compilation [Whaley 2001; Fink and Qian 2003] and is designed for long-running server applications to produce highly efficient code for the best-possible peak performance [Paleczny et al. 2001]. Servers execute mostly long-running applications so that the longer compilation time constitutes only a small total execution time overhead. When compared to the client compiler, the server compiler performs many additional optimizations such as escape analysis, loop invariant code motion, and loop unrolling.

Our trace-based JIT compiler is based on the Java HotSpot client compiler, because the client compiler has a simpler structure and can be modified more easily. Although all techniques are general enough to be also applicable to the server compiler, the complex structure of the server compiler is less approachable for the changes that are required for trace-based compilation, especially in the context of a research project.

Compiling the traces of a trace anchor to optimized machine code involves multiple steps. First, our trace-based compiler builds its HIR by merging the recorded traces into a trace graph. Assuming that the traces in Figure 3(b) were recorded, we build the trace graph shown in Figure 3(d), which is a hybrid between a traditional control flow graph (Figure 3(a)) and a trace tree [Gal and Franz 2006] (Figure 3 (c)) so that both control flow duplication and inner merge nodes are allowed. Merging the traces is explicitly done by the JIT compiler and not during trace recording, because by the 

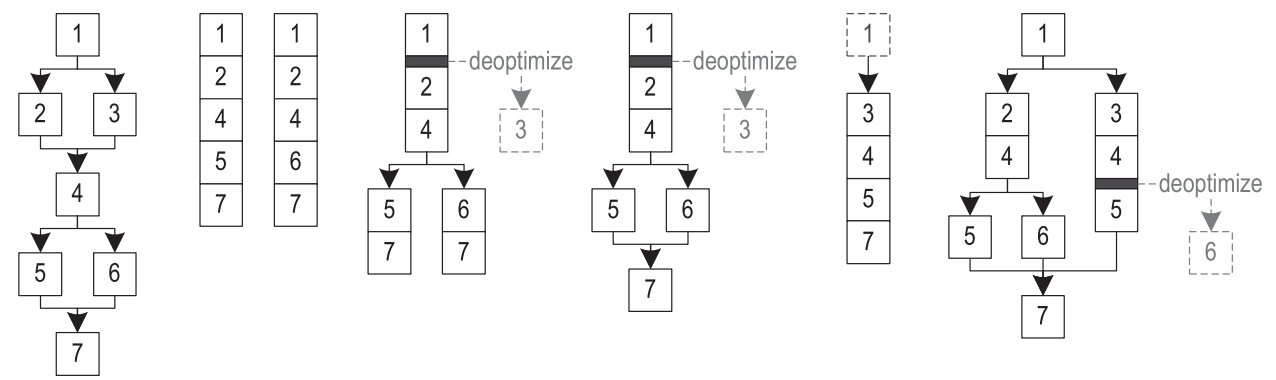

(a) control flow graph

(b) traces

(c) trace tree

(d) initial trace graph

(e) partial trace

(f) final trace graph

Fig. 3. Different high-level intermediate representations and partial traces.

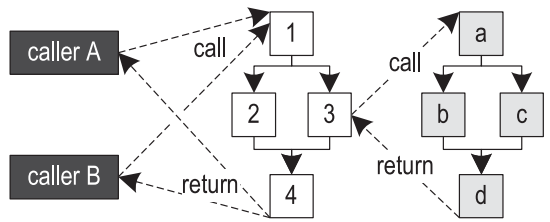

(a) control flow graphs

\section{caller $A \rightarrow 1 \quad 2 \quad 4$}

(b) traces recorded for caller $\mathrm{A}$

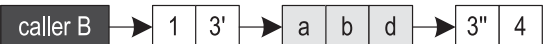

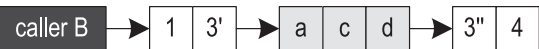

(c) traces recorded for caller B

Fig. 4. Context-sensitive trace information.

time of JIT compilation, all relevant traces have been recorded and more information is available. So, a trace graph allows duplicating code in cases where it makes sense while avoiding code bloat that may occur for trace trees.

On the HIR, we perform general and tracing-specific optimizations such as constant folding and trace inlining. Trace inlining is our most profitable optimization. It is more powerful than method inlining because traces contain context-sensitive information, which helps us to avoid inlining unnecessary method parts [Häubl et al. 2012, 2013]. This is shown in Figure 4, where two different callers invoke the same method. Each caller just needs a certain part of the invoked method so that different traces are recorded for the two callers. When compiling the caller traces, trace inlining is used to inline only those traces that are needed by the specific caller. This is a significant advantage over the context-insensitive profiling information that is typically used by method-based compilers, as such compilers would inline all executed method parts into both callers.

During trace recording, we record type information for the receiver objects of virtual calls. This information is also context sensitive so that our trace-based compiler can inline virtual calls more aggressively without causing code bloat.

In the next compilation step, we generate the LIR that is used for register allocation and code generation. The resulting optimized machine code is then invoked by one of the interpreters or by other compiled traces.

Our trace-based compiler only compiles method parts that are actually covered by traces. Untraced code parts are replaced by deoptimize [Hölzle et al. 1992] instructions 
that fall back to the trace recording interpreter if executed, as shown in Figure 3(d). By omitting untraced code parts, we decrease the number of merge points in the compiled code so that our trace-based compiler can optimize more aggressively. This results in better peak performance and also generates less machine code.

When a deoptimize instruction is executed, we trigger an extended version of the Java HotSpot deoptimization mechanism to fall back to the trace recording interpreter. When execution continues in the trace recording interpreter, a partial trace is recorded that directly starts at the point of deoptimization instead of at the trace anchor; see Figure 3(e). If deoptimization is required too frequently for a piece of machine code, this code is invalidated and recompiled using the originally recorded traces and all partial traces. The additional information in the partial traces allows the compiler to increase method coverage, which in return reduces the deoptimization frequency; see Figure 3(f). The Java HotSpot VM deoptimization mechanism is fairly slow, but it avoids generating compensation code for infrequently executed cases.

\subsection{Compilation Units}

Figure 5(a) shows the call graph for a certain set of methods. Every box represents a method, and arrows are method calls. Within the call graph, method $g$ contains a polymorphic call where three target methods are possible.

Figure 5(b) shows the compilation units for a method-based compiler that uses aggressive method inlining. When method $a$ is getting compiled, the methods $b, c$, and $d$ are inlined, leading to new calls that are inlined again. When the inlining reaches the polymorphic call, the inlining heuristic can either choose to inline none, some, or all target methods. Inlining all target methods can cause significant code bloat so that, for example, the Java HotSpot server compiler only inlines polymorphic calls with a maximum of two target methods. So, the inlining stops at the polymorphic call and each target method is compiled as a separate compilation unit. This example results in four compilation units that have $a, h, i$, and $j$ as their root methods.

Figure 5(c) shows the compilation units of a trace-based compiler that uses aggressive trace inlining. When the method traces of $a$ are getting compiled, the context-sensitive trace information is used for inlining so that when the traces of $f$ are getting inlined into $b$ and $c$, each caller only inlines those traces that it actually requires. The contextsensitive traces and the type of information for the receiver objects of virtual calls help to avoid polymorphic calls. Assume that in this example, the polymorphic call depends on the caller so that only one target method has been observed for every variant of $g$. So, with a sufficiently aggressive inlining policy, the JIT compiler can inline all relevant code parts into one compilation unit.

Figure 5(d) shows possible compilation units for a trace-based compiler that uses less aggressive trace inlining. So, the inlining stops earlier, which results in smaller compilation units so that the method entries of $a, b$, and $i$ become the roots of the resulting three compilation units. However, the context-sensitive trace information still helps to avoid the polymorphic call in this example.

Depending on the size of the resulting compilation units, the JIT compiler can perform different optimizations. Large compilation units offer more opportunities for optimizations, but they also require more time for compilation and more machine code is generated because inlining duplicates code parts. The time required for JIT compilation usually increases more than linearly with the size of the compilation unit as some optimizations have a nonlinear run time. For startup performance, small compilation units are usually better so that the JIT compilation quickly results in machine code that can be executed instead of interpreting the bytecodes. However, for the best peak performance, the compilation units should be sufficiently large to allow good optimizations. 


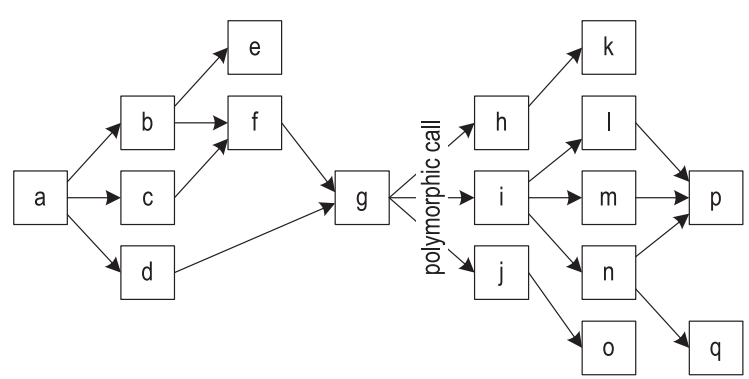

(a) call graph

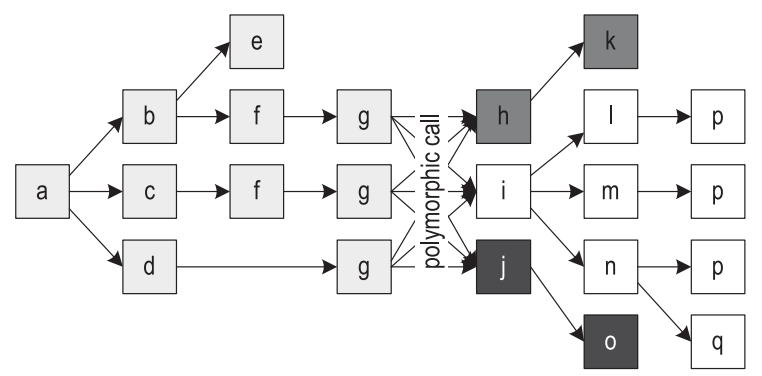

(b) compilation units in a method-based compiler

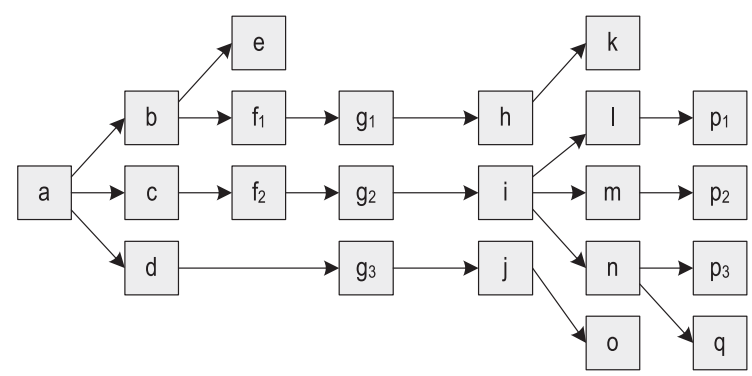

(c) compilation units in a trace-based compiler with aggressive inlining

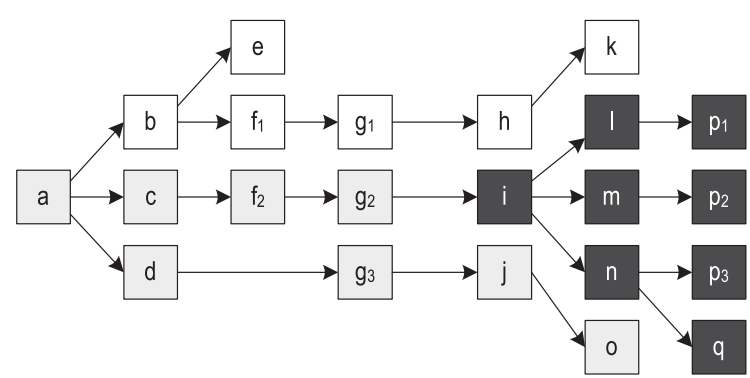

(d) compilation units in a trace-based compiler with less aggressive inlining

Fig. 5. Compilation units. 


\section{TRACE TRANSITIONING}

Unlike other trace-based compilers [Bala et al. 2000; Bebenita et al. 2010b; Gal and Franz 2006; Gal et al. 2006; Chang et al. 2009; Gal et al. 2009; Inoue et al. 2011; Bolz et al. 2009], we do trace inlining during JIT compilation instead of during trace recording [Häubl et al. 2012]. This allows using sophisticated inlining heuristics to avoid unnecessary code bloat as more information is available during JIT compilation. However, it requires a good trace transitioning system as traces may invoke other interpreted or compiled code. So, for our trace-based compiler, invoking traces is as important as invoking methods is for a method-based compiler.

In our previous work [Häubl and Mössenböck 2011; Häubl et al. 2012], we focused on compiling and invoking Java traces that start at method entries. Method traces are fairly simple as there are many similarities to methods so that we were able to reuse large parts of the method invocation mechanisms of the Java HotSpot VM. However, this limited our previous trace transitioning system as compiled code could not invoke interpreted or compiled loop traces. This had a negative effect on the amount of generated machine code and sometimes also resulted in an additional runtime overhead. We extend our previous work with a flexible trace transitioning system that allows arbitrary transitions between interpreted and compiled code.

\subsection{Separating Loops from Methods}

Method-based compilers use On-Stack Replacement (OSR) [Hölzle and Ungar 1994] for long-running loops to switch from interpreted to compiled code in the middle of a method. Which parts of a method are compiled for on-stack replacement is up to the compiler. If the whole method is compiled, the profiling information for the method part before the loop and the method part after the loop is inaccurate as those parts were executed infrequently. This may result in insufficiently optimized machine code. If only the loop is compiled, the optimized code falls back to the interpreter when the loop exits.

When switching from interpreted code to compiled code within a loop, the interpreted stack frame and its values are converted into a compiled stack frame. This conversion can be expensive, but it is done only once so that execution can continue in the compiled machine code. If the loop's parent method is compiled at a later point of execution, this compilation includes the long-running loop so that the OSR machine code will not be executed anymore.

Our trace-based approach is a generalization of OSR. It establishes loops as top-level compilation units to allow complete separation of compiled method traces and compiled loop traces. So, a compiled loop trace has its own stack frame and its own block of machine code that does not necessarily become obsolete when traces of the parent method are compiled. This adds a significant amount of flexibility to our compiler as we can choose to extract loops into their own compilation units when advantageous. It helps us to reduce the size of each compilation unit and affects the compilation time positively as the compilation time often increases more than linearly with the size of the compilation unit.

Invoking loop traces is difficult since many values may flow into loops. Loops can also modify the values that flow into them so that multiple values may have to be returned to the caller when the loop exits. Otherwise, the caller would continue working with outdated values.

To simplify invocation of loop traces, we enforce the following restrictions: we do not compile loop traces separately if they use monitors or operand stack values that were defined outside the loop. So, it is never required to pass monitors or operand stack values to loop traces. Furthermore, we do not compile loop traces separately that 


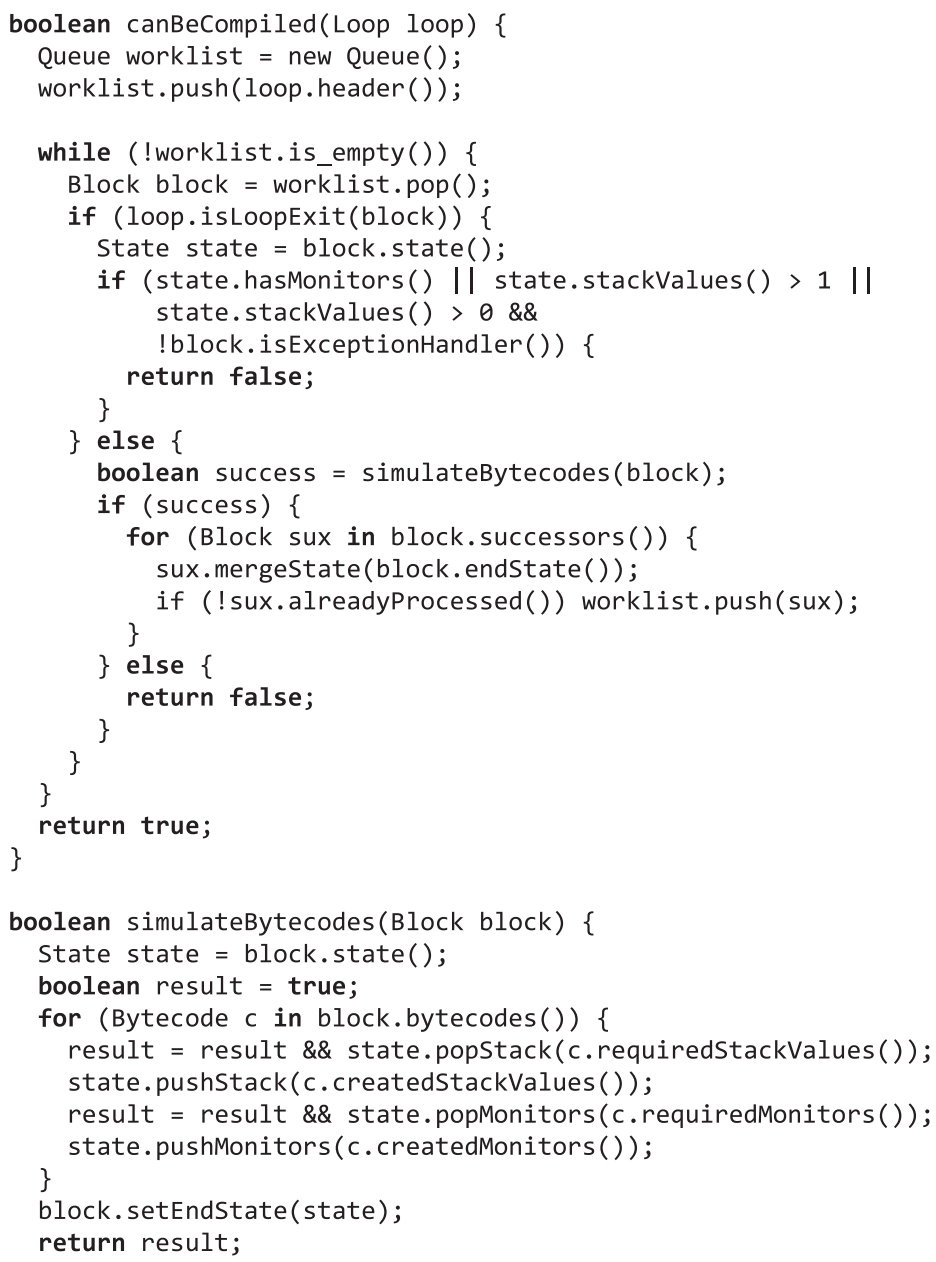

Fig. 6. Bytecode simulation to determine if a loop can be compiled separately.

exit while there are still locked monitors or remaining values on the operand stack. This simplifies returning from loop traces as it is never required to return monitors or operand stack values, while not restricting parameters and local variables that flow into loops.

Both restrictions are small and affect less than $1 \%$ of all loops we have seen so far. To enforce those restrictions, we use abstract bytecode interpretation to simulate how the loop interacts with the operand stack and with monitors. Figure 6 shows that our simulation starts at the loop header with an empty operand stack and without any monitors. During the simulation, we check whether the loop tries to use a nonexisting operand stack value or a nonexisting monitor. If this is the case, the loop traces cannot be compiled separately. Furthermore, we also verify for every loop exit that the operand stack is empty (only exception handlers may have one stack value, which must be the exception object) and that there are no remaining locked monitors. When the simulation ends, the loop is marked as compilable or not compilable according to the simulation 


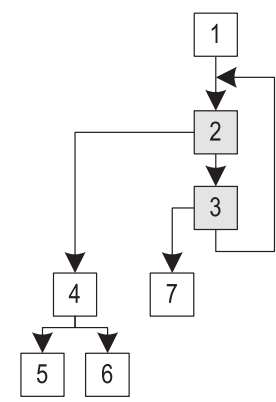

(a) control flow graph

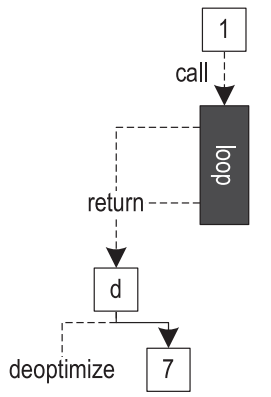

(b) method trace graph

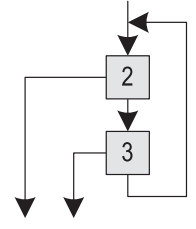

(c) loop trace graph

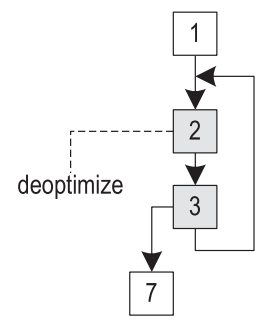

(d) method trace graph with inlined loop trace graph

Fig. 7. Loop invocation.

result. Loops that are not separately compilable have all their traces inlined into their parent trace when the parent trace is compiled.

Figure 7(a) shows the control flow graph of a method that contains a loop, which has the blocks 4 and 7 as its loop exits. A method-based compiler would compile the method as a whole, while our trace-based compiler can compile the loop independently from the remaining method. Figure 7(b) shows a possible trace graph that is created when the recorded method traces are compiled by our trace-based compiler. The loop is invoked by the trace in block 1 and all required values are passed to the loop upon invocation. When the loop exits, it returns all modified values as well as the ByteCode Index (BCI) of the taken loop exit. Execution returns to the artificial block $d$ that uses the returned BCI to dispatch to the correct successor. In the given example, we assume that only block 7 was covered by traces so that execution deoptimizes to the interpreter if the loop exits to block 4.

Figure 7(c) shows the loop trace graph that is built when the recorded loop traces are compiled. This compilation is independent from the compilation of the method traces so that it can occur earlier or later. During its execution, the loop uses the values that were passed upon loop invocation. When a loop exit is encountered, all modified values as well as the BCI of the taken loop exit are returned to the caller.

\subsection{Loop Calling Conventions}

Restricting the way in which loop traces can use monitors and the operand stack greatly simplifies the invocation of loop traces, as it is only necessary to transfer parameters and local variables. Figure 8 shows the calling conventions we use for loop traces. When a loop is invoked, we pass an invocation pointer to an invocation area so that the loop can access the parameters and local variables it actually requires. Figure 8(a) shows that this is simple when the loop is invoked by interpreted code because the parameters and local variables are explicitly contained in the interpreter frame. When the callee loop starts execution, it reads the required values by addressing them relative to the invocation pointer and holds them in registers or spill slots within the loop's stack frame. Eventually, execution reaches a loop exit where it writes back all modified values that the caller might access, again using the invocation pointer as the base address. Finally, the loop returns the BCI of the taken loop exit in a specific register. So, the interpreter can continue execution at the returned BCI since the latest values of parameters and local variables were written back to the interpreter frame.

If the loop is invoked from compiled code, such as in Figure 8(b), the compiled caller holds its values in registers or spill slots. So, the caller allocates an invocation area 


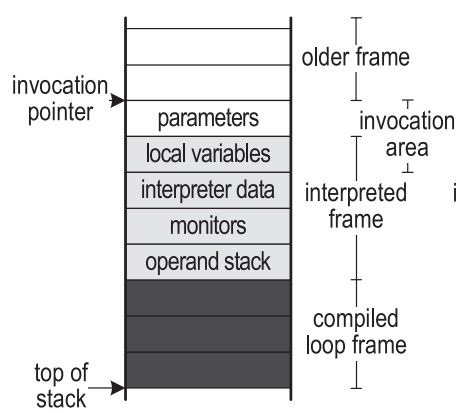

(a) interpreted method invokes compiled loop

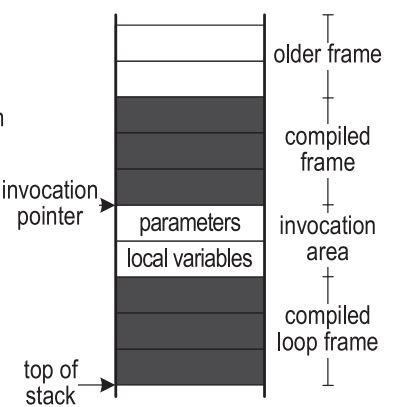

(b) compiled code invokes compiled loop

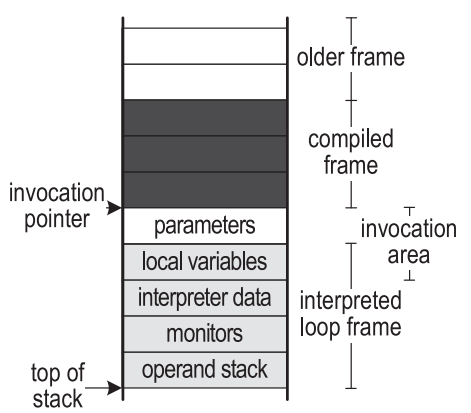

(c) compiled code invokes interpreted loop

Fig. 8. Calling conventions for loop traces.

at the top of its compiled frame, where it spills its live parameters and local variables before invoking the loop. This brings the parameters and local variables into a defined order and allows the caller to pass an invocation pointer to the callee. The callee loop reads the required values upon loop entry and writes back all modified values when the loop exits. The BCI of the taken loop exit is then returned in a specific register so that the caller can determine where to continue execution. If the invoked loop has only one loop exit, the caller can continue directly without checking the returned BCI. However, if the invoked loop has more than one exit, the compiled caller uses the returned BCI to dispatch to the correct location in the compiled code. Before execution actually continues, it first reads the latest values from the invocation area.

Our calling conventions also support calling an interpreted loop from compiled code, as shown in Figure 8(c). This allows the calling traces to be compiled earlier than the loop traces. At loop invocation, the compiled caller spills the live parameters and local variables to the invocation area at the top of its frame. Because the parameters and local variables are in the same order as in an interpreter frame, the interpreter just needs to allocate a partial stack frame containing space for the interpreter-specific data, monitors, and operand stack. So, the invocation area becomes a part of the interpreter frame. During loop execution, the interpreter modifies the parameters and local variables in place. When the loop exits, the interpreter-specific part of the stack frame is discarded and the loop exit BCI is returned in a specific register. Because the parameters and local variables were modified in place, the invocation area already contains the latest values so that no data has to be written back explicitly. The compiled caller then continues execution as if a compiled loop were invoked.

The case shown in Figure 8(c) also occurs after deoptimization of a compiled loop trace that was invoked by compiled code. After deoptimization, the stack looks exactly as if the compiled code invoked an interpreted loop. If this calling convention variant was not supported, the compiled caller would have to be deoptimized as well, which could result in cascading deoptimizations. By giving the deoptimized loop a separate interpreter frame, we can isolate the effect of deoptimization so that it affects just a single stack frame.

Figure 9 shows that our calling conventions enable arbitrary transitions between interpreted and compiled traces. This was not supported by our previous work and adds a great amount of flexibility to our system. The transitions can be grouped into the following four categories:

(1) The first category covers transitions from interpreted to interpreted code. Those are mainly relevant during startup when the application is executed in the 


\begin{tabular}{|c|c|c|c|c|}
\hline from frame & $\begin{array}{l}\text { interpreted method traces } \\
\text { infrequently executed code } \\
\text { or after deoptimization }\end{array}$ & $\begin{array}{c}\text { interpreted loop traces } \\
\text { infrequently executed } \\
\text { code }\end{array}$ & $\begin{array}{l}\text { compiled method traces } \\
\text { frequently executed code }\end{array}$ & $\begin{array}{c}\text { compiled loop traces } \\
\text { frequently executed } \\
\text { code }\end{array}$ \\
\hline $\begin{array}{l}\text { interpreted method traces } \\
\text { infrequently executed code } \\
\text { or after deoptimization }\end{array}$ & yes & same frame & yes & yes \\
\hline \begin{tabular}{|l|} 
interpreted loop traces \\
after deoptimizing a \\
compiled loop or invoking an \\
infrequently executed loop \\
from compiled code
\end{tabular} & yes & same frame & yes & yes \\
\hline $\begin{array}{l}\text { compiled method traces } \\
\text { frequently executed code }\end{array}$ & yes & yes & yes & yes \\
\hline $\begin{array}{l}\text { compiled loop traces } \\
\text { frequently executed code }\end{array}$ & yes & yes & yes & yes \\
\hline
\end{tabular}

Fig. 9. Transitions between interpreted and compiled traces.

interpreter and hardly any code has been compiled yet. In later execution phases, those transitions are still required because the compiled code may deoptimize to the interpreter.

(2) The second category covers transitions from compiled code to interpreted code. Those transitions occur when frequently executed traces are compiled and less-hot traces are inlined during JIT compilation. Then, those less-hot traces may invoke other code that was not jitted yet.

(3) The third category covers transitions from interpreted code to compiled code, which occurs during startup when not-yet-compiled code invokes code that was already jitted. This happens because more frequently executed code (e.g., code with multiple callers) is hotter and therefore compiled earlier. After startup, those transitions still occur when the caller was deoptimized and is therefore interpreted.

(4) The fourth category covers transitions from compiled to compiled code. In nontrivial applications, those transitions occur most frequently because all relevant code parts are jitted eventually. So, to achieve a good peak performance, most trace transitions must fall into this category.

In comparison to that, Figure 10 shows the transitions possible in a traditional method-based system. There, loops are always compiled with their enclosing method and do not have separate stack frames.

We also evaluated a concept for register-based loop calling conventions. Such calling conventions could be more efficient and could decrease the loop invocation overhead but would significantly complicate the transition between interpreted and compiled code. Because the loop body is usually executed multiple times, execution tends to stay within a called loop for a longer time than within a called method. Furthermore, for best peak performance, it is more profitable to inline the loop, as mentioned in Section 3.3. So, it is unlikely that peak performance would profit significantly from register-based calling conventions for loop traces.

\subsection{Loop Inlining}

Method-based JIT compilers compile whole methods (including loops) to optimized machine code. During compilation, the compiler collects information about the values that are used within the method and the loops. This information may consist of types, constant values, or the observation that an object does not escape the method. Common 


\begin{tabular}{|l|c|c|c|c|}
\hline \multicolumn{1}{|c|}{ to frame } & $\begin{array}{c}\text { interpreted method } \\
\text { infrequently executed code } \\
\text { or after deoptimization }\end{array}$ & $\begin{array}{c}\text { interpreted loop } \\
\text { infrequently executed } \\
\text { code }\end{array}$ & $\begin{array}{c}\text { compiled method } \\
\text { frequently executed code }\end{array}$ & $\begin{array}{c}\text { compiled loop } \\
\text { frequently executed } \\
\text { code }\end{array}$ \\
\hline $\begin{array}{l}\text { interpreted method } \\
\text { infrequently executed code } \\
\text { or after deoptimization }\end{array}$ & yes & same frame & yes \\
\hline interpreted loop & \multicolumn{3}{|c|}{ interpreted loops never have a separate stack frame } \\
\hline $\begin{array}{l}\text { compiled method } \\
\text { frequently executed code }\end{array}$ & yes & impossible & yes & same frame \\
\hline compiled loop & \multicolumn{3}{|c|}{ compiled loops never have a separate stack frame } \\
\hline
\end{tabular}

Fig. 10. Transitions between interpreted and compiled methods.

optimizations such as inlining of polymorphic calls or constant folding make use of this information. If a loop and its caller are compiled together, this information can be used for optimizations in the loop. However, if the loop is compiled separately before its caller, the following problems occur:

- The invoked loop does not have much information about the incoming values. As a large number of values may flow into a loop, this lack of information can preclude optimizations in the invoked loop.

- The loop caller has the problem that a loop invocation kills all information about the values that could be modified by the loop. This may preclude optimizations in the caller and is a significant problem for nested loops, where the inner and the outer loops operate on the same values.

The significance of those issues can be reduced by recording profiling information about the passed values. To mitigate all disadvantages, trace-based compilation must perform loop inlining. For this, the trace-based compiler builds the loop's trace graph and replaces the loop invocation with that graph, as shown in Figure 7(d). Then, all loop exits are linked to their correct successor blocks using jump instructions. This allows the compiler to optimize the loop and its caller as a whole, which results in a similar effect as method inlining.

In our previous work [Häubl et al. 2012], loops were always inlined as we had not yet defined suitable calling conventions for loops. This had a negative impact on the startup performance and the size of the generated machine code as loop inlining makes the code of the separately compiled loop obsolete. Our new trace-based compiler uses the recorded probability of entering the loop to decide if the loop should be compiled separately or if it should be inlined.

\section{EXCEPTION HANDLING}

Although exceptions should only be thrown in rare cases, some Java applications heavily rely on exception handling. A few applications even use exception handling for control flow decisions. Therefore, a trace-based JIT compiler also must support the most frequent exception handling cases in an efficient way.

Exception handling in method-based compilers always either continues directly in the exception handler or unwinds the call stack and handles the exception in one of the callers. When a method frame is unwound, all values that were used within the method are discarded as they are no longer needed. For a trace-based compiler, this is more difficult. If an exception is thrown in a loop and caught in the loop's parent, any values that were modified in the loop have to be returned to the parent when the loop frame is discarded. This is necessary so that execution can continue in the exception handler using the latest values. 


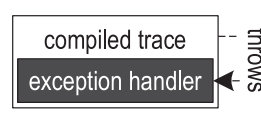

(a) exception within a compiled trace

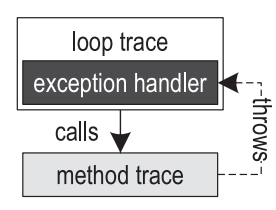

(b) exception unwinds from a method trace

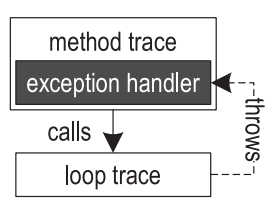

(c) exception unwinds from a loop trace

Fig. 11. Exception handling.

Previous trace-based compilers for Java [Bebenita et al. 2010b; Gal and Franz 2006; Gal et al. 2006; Inoue et al. 2011] either stopped or aborted trace recording when an exception was thrown. This simplifies trace recording but may result in inefficient exception handling when the traces are compiled to machine code. Our trace recording approach supports exceptional control flow so that exception sources and exception handlers frequently are within the same compilation unit, as shown in Figure 11(a). If the exception handler was covered by recorded traces and was therefore compiled to machine code, exception handling is as efficient as possible because the compiled code can dispatch directly to the exception handler. If the exception handler was not compiled, execution deoptimizes to the interpreter. Because a trace-based compiler tends to have larger compilation units than a method-based compiler (traces are smaller than methods and therefore have a higher potential to be inlined), exceptions stay within the same compilation unit most of the time.

Figure 11(b) shows the case where an exception unwinds from a method trace and execution continues within a loop trace. When unwinding the method trace, all live values can be discarded as they are not needed by the caller. In that case, our exception handling is similar to exception handling in a method-based compiler. The same approach is also taken when a loop trace throws an exception that is caught neither within the loop trace nor within its enclosing method.

Figure 11(c) shows the case where an exception unwinds from a loop trace and execution continues in the trace of its enclosing method. The loop trace might have modified some parameters or local variables, so execution must use the latest values when it continues in the exception handler. This case is complicated by the fact that the involved frames can be either interpreted or compiled.

When a compiled loop trace throws an exception that is caught in its enclosing method that was compiled separately, we deoptimize the loop trace to the interpreter. This creates the case shown in Figure 8(c) and ensures that the loop's latest values are written back to the invocation area of the caller. Then, the frame of the loop trace is unwound and exception handling continues in the caller. The caller loads the latest values and rethrows the exception using the BCI of the instruction where the exception initially occurred in the loop trace. Eventually, execution continues in the correct exception handler with the latest values.

If this case occurs for an interpreted loop that was invoked by compiled code, we immediately unwind the interpreter-specific part of the loop frame because the interpreter modified the invocation area in place. So, the invocation area already contains the latest values and exception handling can directly continue in the caller.

If an exception unwinds from a loop trace and the exception is caught in the enclosing method, our approach has some overhead as it relies on deoptimization. This overhead is only relevant for frequently thrown exceptions and can be addressed easily using loop inlining so that exceptions are thrown within the same compilation unit, which is the most efficient way to handle exceptions. 


\section{FURTHER OPTIMIZATIONS}

In addition to our calling conventions and the better exception handling, we added some more optimizations to our trace-based compiler. We added a type system that can store different kinds of type information for every SSA value because the original type system of the Java HotSpot client compiler was fairly limited. Our new type system is especially useful for inlining of polymorphic calls, where the receiver is represented by a single SSA value but its type may vary for every inlined method. Furthermore, it is also possible to keep track of the fact that the type of value is a subtype of a specific class or that a value has multiple possible types.

Our new trace-based compiler also incorporates a more advanced trace inlining technique that we described in a recently accepted article [Häubl et al. 2013]. The work in that article significantly increased peak performance but focused on trace inlining heuristics and covered neither trace transitioning nor exception handling.

\subsection{Type-Specific Optimizations}

Figure 12(a) shows the Java source code of the method concat (), which concatenates two character sequences. Two call sites that invoke concat () with different parameters are shown in Figure 12(b). When the callers are getting compiled, each caller inlines those concat () traces that were recorded for the specific caller. This context sensitivity allows specializing the inlined code for the specific caller and reduces the number of types observed for each value so that aggressive type-specific optimizations can be used more frequently.

The first caller only passes String objects to concat(), which is also reflected by the type information recorded in the traces. When inlining those traces, the JIT compiler generates the optimized pseudo-code shown in Figure 12(c) and uses runtime checks to guard the assumption that only String objects are passed as $a$ and $b$. When such a runtime check fails, the execution deoptimizes to the trace recording interpreter.

For the remaining compilation unit, the compiler knows that $a$ and $b$ are always String objects so that it is possible to change the CharSequence.length() interface calls to String. length() calls. Unlike the interface calls, those calls can be inlined easily. Furthermore, it is now also possible to eliminate the instance-of checks as neither $a$ nor $b$ is a StringBuffer object. So, it is known that result is a StringBuilder object so that the calls to append() can also be inlined easily.

The second caller either passed a String or a StringBuilder object as the first parameter and always used a String object as the second parameter. The optimized pseudocode for this caller is shown in Figure 12(d). The first call to CharSequence.length() is polymorphic as the type information in the traces indicates that $a$ is either a String or a StringBuilder. So, two type checks are added that dispatch to the inlined versions of String. length() and StringBuilder.length(). If $a$ is neither a String nor a StringBuilder, the execution deoptimizes to the trace recording interpreter. For the invocation of CharSequence. length () on $b$, a guard is added to ensure that $b$ is a String object. In the next step, it is possible to eliminate the instance-of checks as neither $a$ nor $b$ is a StringBuffer. So, the result is a StringBuilder object and the calls to append() can be inlined.

By using the type information for optimizations such as removal of type checks and elimination of redundant guards, we increase the performance and help the compiler to reduce the bookkeeping information that is required for deoptimization.

\subsection{Tail Duplication}

One optimization typical for trace-based compilers is tail duplication, which duplicates control flow along frequently executed paths [Bala et al. 2000]. If performed excessively, 


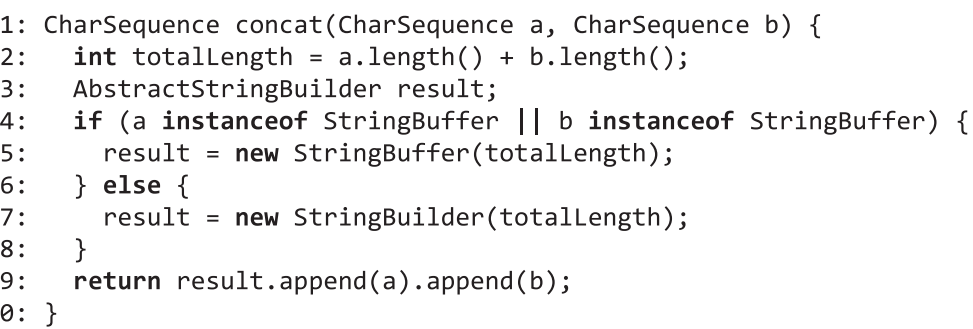

(a) concatenation source code

A concat (string, string);
B concat (stringorstringBuilder, string);

(b) concatenation callers

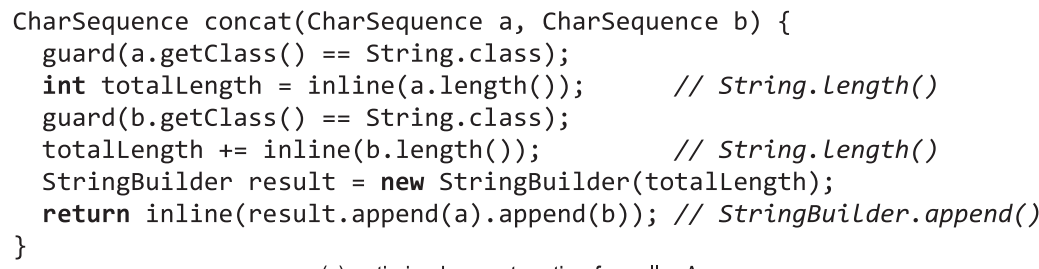

(c) optimized concatenation for caller $\mathrm{A}$

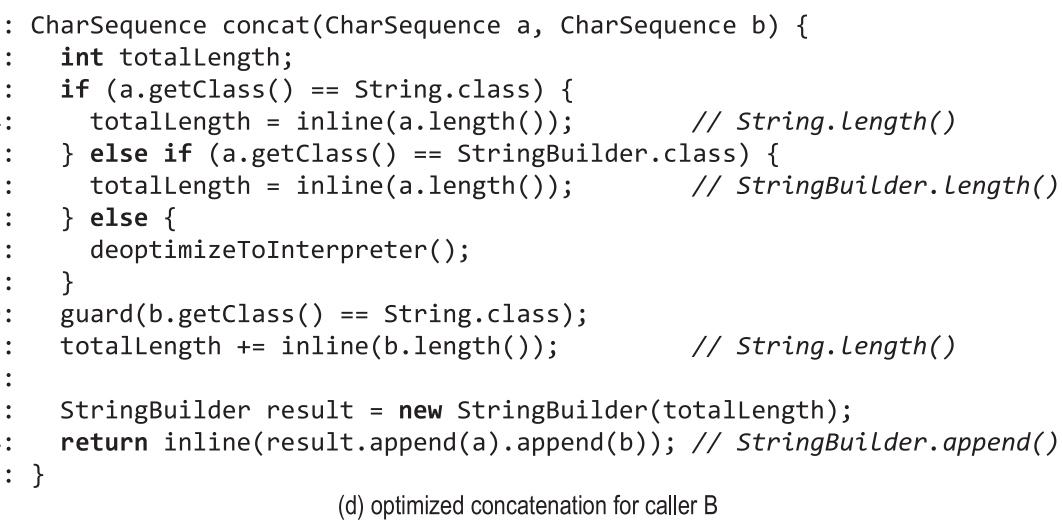

(d) optimized concatenation for caller B

Fig. 12. Type-specific optimizations.

this results in a trace tree [Gal et al. 2006] without any inner merge nodes but also exponential growth of compiled code because of the duplicated control flow. To avoid excessive tail duplication, we merge multiple traces into a trace graph prior to compilation. This data structure is a hybrid between a control flow graph and a trace tree and allows both control flow duplication and inner merge nodes.

Combining tail duplication and aggressive trace inlining can easily result in code bloat because calls may get inlined multiple times when the call itself is duplicated. Therefore, we use a heuristic that ensures that only small blocks (up to 20 bytecodes) are duplicated. When a larger block is encountered, we merge the control flow so that the block is only compiled once. However, a successive small block can again trigger control flow duplication. We also experimented with larger amounts of tail duplication, but we 
were not able to achieve a higher peak performance, while the amount of generated machine code and the time required for JIT compilation increased dramatically.

\section{EVALUATION}

Our trace-based JIT compiler was implemented for the IA-32 architecture of Oracle's Java HotSpot VM using the early access version b12 of the upcoming JDK 8 [Oracle Corporation 2013]. For benchmarks, we use an Intel Core-i5 processor with four cores running at $2.66 \mathrm{GHz}, 4 * 256 \mathrm{kB} \mathrm{L} 2$ cache, $8 \mathrm{MB}$ shared L3 cache, $8 \mathrm{~GB}$ main memory, and Windows 8 Professional operating system. We evaluate our trace-based compiler on the benchmark suites SPECjvm2008 [Standard Performance Evaluation Corporation 2008] and DaCapo 9.12 Bach [Blackburn et al. 2006] and present results for the following configurations:

- The configuration HotSpot client uses the unmodified method-based Java HotSpot client compiler from the early access version b12 of the upcoming JDK 8. This is our baseline as our trace-based JIT compiler builds on the client compiler. All other results are normalized to results of this configuration, which is only implicitly shown in the figures as the $100 \%$ mark.

- The configuration tracing peak performance represents our trace-based JIT compiler and uses aggressive trace inlining and all exception handling mechanisms described in this article. It is optimized for peak performance while generating still reasonable amounts of machine code. Furthermore, for nested loops, we ensure that inner loops are at least inlined into the outermost loop of the same method. This increases the performance in case the inner and the outer loops operate on the same values, as the loops are optimized as one compilation unit.

- The configuration tracing minimum code also represents our trace-based JIT compiler and uses all exception handling mechanisms described in this article. However, less aggressive trace inlining is used so that loop traces are less frequently inlined and more often compiled separately. This decreases the amount of generated machine code while not sacrificing too much peak performance.

- The configuration tracing abort on exception is optimized for peak performance but aims to illustrate how important good exception handling is for the peak performance of complex applications. So, it aborts and invalidates the record traces in case an exception is thrown. This is the simplest way of handling exceptions (e.g., used by Gal and Franz [2006], Gal et al. [2006], and Bebenita et al. [2010b]) but has the disadvantage that those instructions that always throw an exception are never compiled. We also tried another variant that stops recording the current trace when an exception is thrown so that the instruction that throws the exception and the corresponding exception handler are never within the same compilation unit. This approach was used, for example, by Bebenita et al. [2010a] and Inoue et al. [2011] but shows almost the identical performance as aborting the traces.

-The configuration HotSpot server represents the unmodified, method-based Java HotSpot server compiler from the early access version b12 of the upcoming JDK 8. This JIT compiler is explicitly designed for best peak performance and performs significantly more optimizations than our trace-based compiler (e.g., escape analysis and sophisticated loop optimizations).

We evaluate our compiler with the SPECjvm2008 and the DaCapo 9.12 Bach benchmark suites. Each benchmark suite is measured in terms of peak performance, amount of generated machine code, and compilation time. To ensure comparison fairness, all presented configurations use the same garbage collector. For our trace-based compiler, the amount of generated machine code also includes data specific to trace-based compilation such as additional deoptimization information required for fall-back to the 


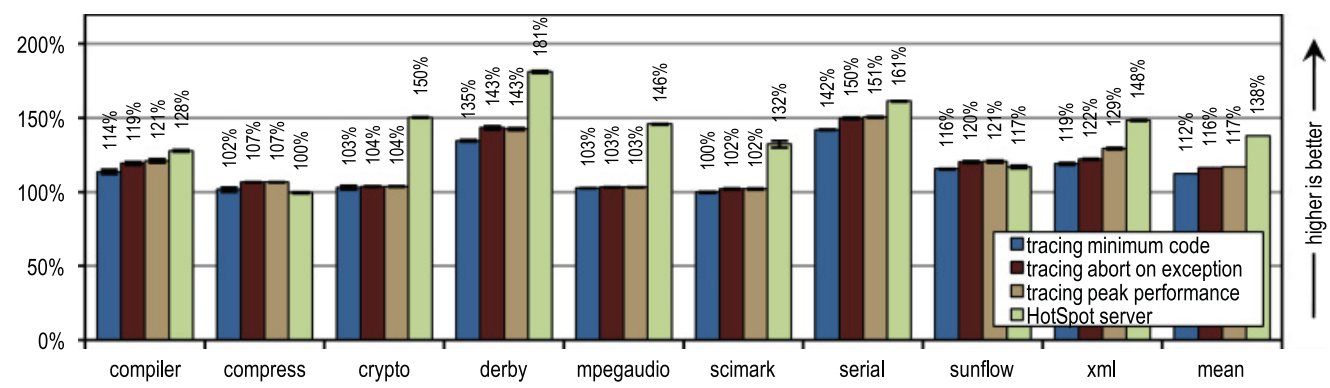

Fig. 13. SPECjvm2008: peak performance.

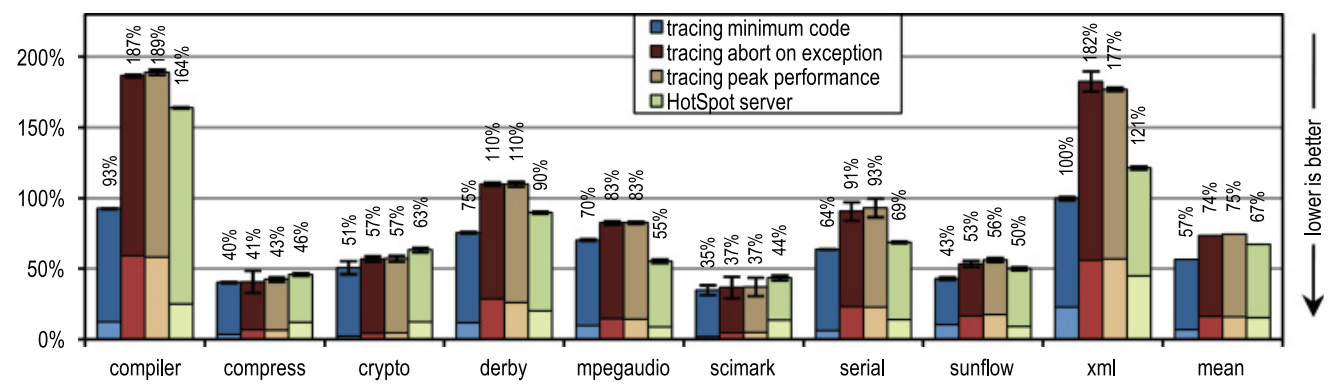

Fig. 14. SPECjvm2008: generated machine code.

interpreter. Each benchmark suite was executed 15 times and we report the average of those results along with the $95 \%$ confidence interval.

\subsection{SPECjvm2008}

The SPECjvm2008 benchmark suite consists of nine benchmark categories that measure the peak performance. Next to the individual benchmark results, we present the geometric mean of all results. A heap size of 1,024MB is used for all measurements.

Figure 13 shows that all our trace-based configurations outperform the client compiler in all cases. On the benchmarks compress and sunflow, our configuration tracing peak performance slightly outperforms the Java HotSpot server compiler. This is mainly due to the aggressive trace inlining, but also, the type system with the contextsensitive information has some impact. In general, our tracing configurations achieve a peak performance that is somewhere between the Java HotSpot client and the HotSpot server compiler. On average, the speedup to the client compiler is $17 \%$ with aggressive inlining and $12 \%$ with moderate inlining.

For rather small and loop-intensive benchmarks such as crypto, mpegaudio, and scimark, we achieve only a small speedup compared to the client compiler. This is the case because our trace-based compiler does not perform any sophisticated loop optimizations yet. Furthermore, trace inlining does not have a significant advantage over method inlining on small and loop-intensive benchmarks because a method-based compiler can also inline all relevant code parts [Häubl et al. 2012]. The SPECjvm2008 benchmarks also hardly throw any exceptions, so changing the exception handling has a modest impact only on the benchmarks compiler and $\mathrm{xml}$.

Figure 14 shows the amount of generated and invalidated machine code on top of each other. The dark bars in Figure 14 show the total amount of generated machine code, while the light bars indicate the amount of machine code that was invalidated because optimistic optimizations deoptimized too frequently. The figure shows that our 


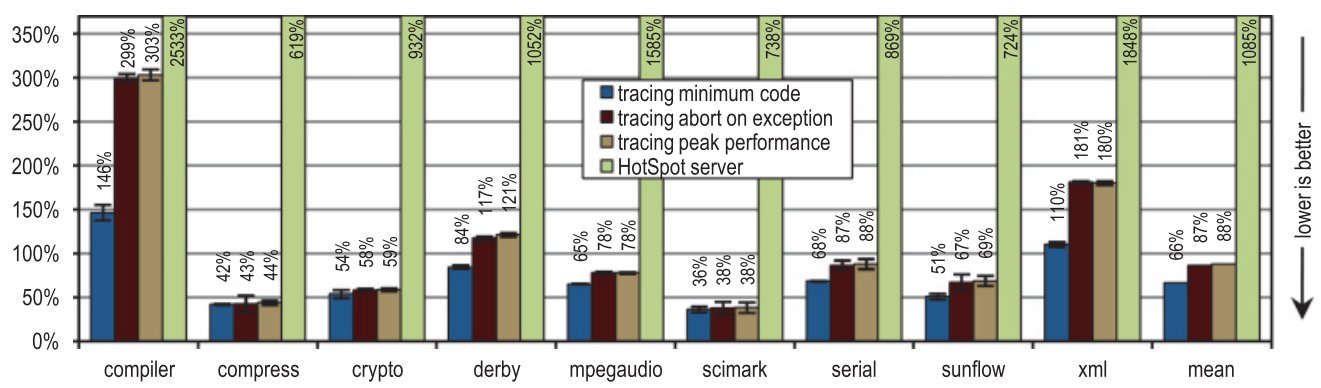

Fig. 15. SPECjvm2008: time required for JIT compilation.

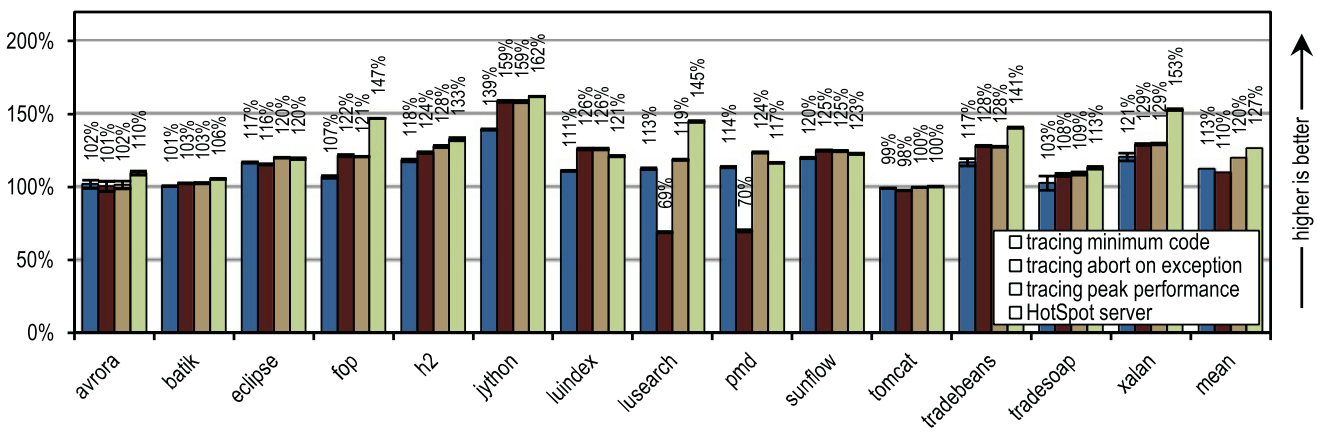

Fig. 16. DaCapo 9.12 Bach: peak performance.

tracing configurations generate less machine code than the client compiler on average. Especially, our configuration tracing minimum code is efficient in terms of generated machine code. For the benchmarks compiler and $x \mathrm{ml}$, some of our tracing configurations use too aggressive optimizations during startup, so that the overspecialized code deoptimizes frequently and must be recompiled later on, which results in a fairly high amount of generated machine code. The configuration tracing abort on exception shows that good exception handling hardly increases the size of the generated machine code.

Figure 15 shows the time required for JIT compilation. On average, our tracing configuration tracing minimum code spends $34 \%$ less time on JIT compilation than the client compiler, while our configuration tracing peak performance still needs $12 \%$ less time than the client compiler. The server compiler, which is explicitly designed for peak performance, and which performs significantly more optimizations than our trace-based compilers, spends more than $12 \mathrm{x}$ as much time on JIT compilation than our configuration tracing peak performance.

\subsection{DaCapo}

The DaCapo 9.12 Bach benchmark suite consists of 14 Java applications. Each benchmark is executed 20 times with the default data size so that the execution time converges. Next to the individual benchmark results, we present the geometric mean of all results. A heap size of $1,024 \mathrm{MB}$ is used for all measurements.

Figure 16 shows the peak performance results for the DaCapo 9.12 Bach benchmark suite. Several of the DaCapo 9.12 Bach benchmarks use exceptions so that the bad exception handling of the configuration tracing abort on exception significantly affects the performance. Compared to the client compiler, our configurations tracing minimum code and tracing peak performance show a similar or higher peak performance on all benchmarks. For the benchmarks luindex, pmd, and sunflow, our configuration 


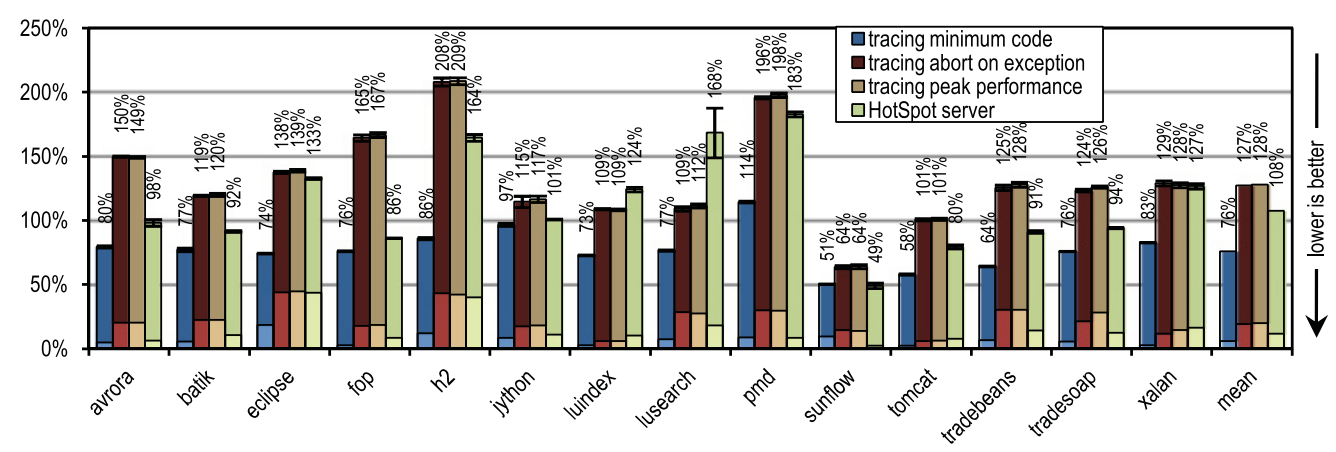

Fig. 17. DaCapo 9.12 Bach: generated machine code.

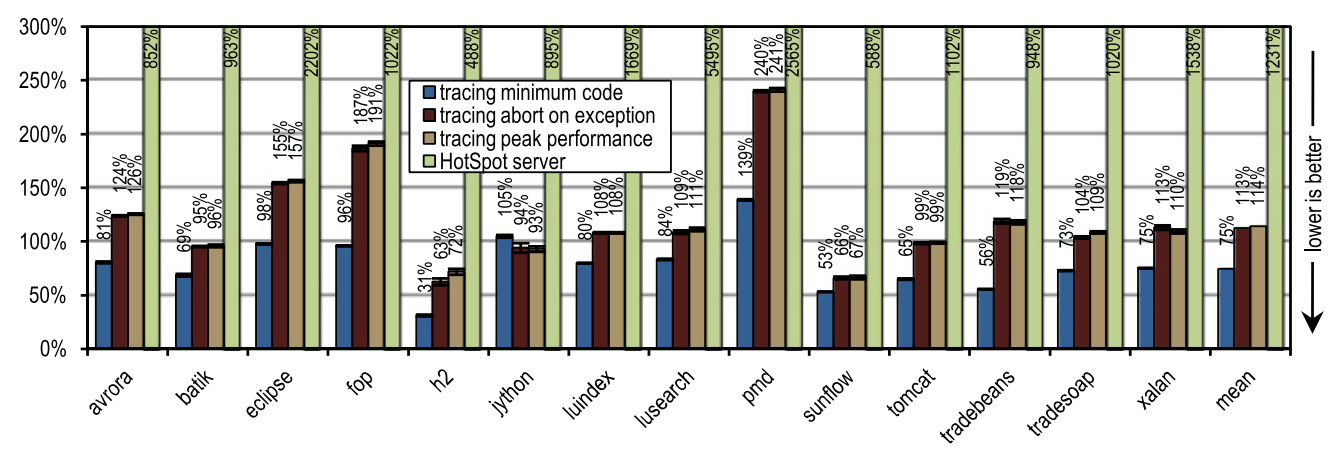

Fig. 18. DaCapo 9.12 Bach: time required for JIT compilation.

tracing peak performance outperforms the server compiler. The benchmarks luindex and sunflow profit from the aggressive trace inlining and the context-sensitive type information, while the high performance of $p m d$ is the result of trace inlining and the good exception handling of our trace-based compiler.

In general, our tracing configurations achieve a peak performance between the client and the server compiler, where tracing peak performance is on average $20 \%$ faster than the client compiler and only 6\% behind the server compiler. Compared to the client compiler, we achieve the highest speedup on the benchmark jython, which executes a large number of virtual calls that can be inlined by our compiler. This benchmark also profits from trace inlining and the larger compilation units of our trace-based approach.

The dark bars in Figure 17 show the total amount of generated machine code, while the light bars indicate the amount of machine code that was invalidated because optimistic optimizations deoptimized too frequently. Our configuration tracing minimum code generates less machine code than the client compiler, while the configuration tracing peak performance generates more. The configuration tracing abort on exception shows that not compiling exception throwing bytecodes hardly reduces the size of the generated machine code.

Figure 18 shows that our configuration tracing minimum code requires $25 \%$ less time for JIT compilation than the client compiler, while tracing peak performance requires $14 \%$ more time than the client compiler. The server compiler performs significantly more optimizations and spends more than $12 \mathrm{x}$ as much time on JIT compilation than the client compiler. 


\begin{tabular}{|l|c|c|c|c|}
\hline \multicolumn{1}{|c|}{ to frame } & $\begin{array}{c}\text { interpreted } \\
\text { method traces }\end{array}$ & $\begin{array}{c}\text { interpreted } \\
\text { loop traces }\end{array}$ & $\begin{array}{c}\text { compiled } \\
\text { method traces }\end{array}$ & $\begin{array}{c}\text { compiled } \\
\text { loop traces }\end{array}$ \\
\hline interpreted method traces & 4923 & same frame & 8197 & 357 \\
\hline interpreted loop traces & 10 & same frame & 164 & 1 \\
\hline compiled method traces & 720 & 69 & 1113093 & 49806 \\
\hline compiled loop traces & 78 & 8 & 667908 & 43432 \\
\hline
\end{tabular}

Fig. 19. Transition frequencies in thousands for the SPECjvm2008 benchmarks.

\begin{tabular}{|l|c|c|c|c|}
\hline \multicolumn{1}{|c|}{ to frame } & $\begin{array}{c}\text { interpreted } \\
\text { method traces }\end{array}$ & $\begin{array}{c}\text { interpreted } \\
\text { loop traces }\end{array}$ & $\begin{array}{c}\text { compiled } \\
\text { method traces }\end{array}$ & $\begin{array}{c}\text { compiled } \\
\text { loop traces }\end{array}$ \\
\hline interpreted method traces & 13366 & same frame & 50637 & 522 \\
\hline interpreted loop traces & 52 & same frame & 730 & 1 \\
\hline compiled method traces & 2616 & 350 & 1119335 & 46805 \\
\hline compiled loop traces & 267 & 36 & 290831 & 1319 \\
\hline
\end{tabular}

Fig. 20. Transition frequencies in thousands for the DaCapo 9.12 Bach benchmarks.

\subsection{Discussion of Results}

Our tracing configurations perform especially well on large applications such as the DaCapo 9.12 Bach benchmarks, where aggressive trace inlining results in large compilation units and better-optimized machine code. Combined with the good exception handling and optimizations that use the context-sensitive type information, our configuration tracing peak performance outperforms the server compiler on a few benchmarks. This is interesting as the server compiler is designed for best peak performance and implements significantly more optimizations than our trace-based compiler. Our results show that a fairly simple trace-based compiler can achieve an excellent peak performance by performing only basic traditional optimizations and aggressive trace inlining. Depending on the target platform, our configuration tracing minimum code is also interesting as it combines a good peak performance with little generated machine code and an excellent compilation time.

For some applications and benchmarks, such as DaCapo 9.12 Bach lusearch and pmd, good exception handling is crucial. Ignoring exception handling during trace recording, as is done in most of the related work, leads to unacceptable slowdowns for some applications and is therefore not suitable for production use. Our tracing of thrown exceptions and our trace inlining approach ensure that frequently thrown exceptions are compiled together with the corresponding exception handler. This significantly increases the performance of benchmarks with lots of exception handling.

Our trace-based compiler does not do loop optimizations such as loop unrolling or loop-invariant code motion, so there is only a small performance gain for small and loop-intensive benchmarks. There, the server compiler is especially strong as it does perform sophisticated loop optimizations. However, this also shows that our trace-based compiler still has plenty of potential as it performs only basic traditional optimizations so far.

Figures 19 and 20 show the average trace transition frequencies for the benchmark suites and the configuration tracing minimum code. The largest number are transitions from compiled code to compiled code, which is the fourth category according to Figure 9. This occurs because all relevant code parts have been compiled when the application reaches its peak performance. Furthermore, the number of transitions to compiled method traces is significantly larger than the number of transitions to compiled loop traces, for two reasons. First, compiled loops are invoked less frequently as execution stays within loops for a longer time than within methods because the loop body is 
typically executed multiple times. To directly compare those two numbers, the loop invocations would have to be scaled by the average number of loop iterations. Second, it is disadvantageous to focus solely on compiling loops as aggressive trace inlining might result in huge compilation units before inlining ends because of reaching the next loop. Furthermore, the compiler would still lack information about the values that flow into the loop because those values are passed in from the enclosing method. When compared to our previous work [Häubl et al. 2012], where loops were always inlined, the startup performance and the size of the generated machine code both improved by $2 \%$ on average.

\section{RELATED WORK}

Our trace-based compiler is a hybrid between a tracing compiler [Bala et al. 2000] and a traditional method-based compiler that uses partial-method compilation [Whaley 2001; Fink and Qian 2003]. We compare our contribution against both trace-based and method-based related works.

\subsection{Trace-Based Compilation}

Trace compilation was pioneered by Bala et al. [2000] in their Dynamo system. They used a software interpreter to execute native instruction streams. During execution, hot instruction sequences are identified and recorded. Those traces are then optimized and the generated machine code is executed directly. The Dynamo system assumes that every backward branch is a possible loop header. However, this leads to the detection of false loops [Hayashizaki et al. 2011].

Trace-based compilation is also used for compiling dynamically typed languages such as ActionScript [Chang et al. 2009], JavaScript [Gal et al. 2009], or Python [Bolz et al. 2009]. In those cases, the recorded traces contain information about the encountered types so that the trace-based compiler can perform type specialization. This results in high speedups as boxed types can be replaced by primitive types so that operations such as adding two integers can be replaced by a single hardware instruction instead of a method call. In contrast to that, Java is a statically typed language so that most type information is already available at compile time. Therefore, similar optimizations are mostly not applicable for Java. We focus on work that is targeting Java, as this is closest to our work.

Rogers [2002] implemented a JIT compiler for Java that detects and compiles frequently executed basic blocks. Frequently executed blocks that are related to each other are grouped together, optimized, and compiled to machine code. In comparison to method-based compilation, this approach compiles up to $18 \%$ fewer bytecodes.

Gal and Franz [2006] and Gal et al. [2006] used trace-based compilation to compile Java on resource-constrained devices. Similar to the Dynamo system, backward branch targets and side exits of existing traces are considered as trace anchors. When a trace anchor was executed frequently enough, trace recording is started and the recorded trace is compiled to machine code. If an exception occurs during trace recording, they abort trace recording and invalidate the recorded traces. Compiled traces are linked together to form a trace tree with explicit tail duplication. While the trace tree simplifies many optimizations, it cannot be used for applications with a complex control flow because excessive tail duplication results in code bloat. Still, this approach is fairly popular and a similar concept is used by the Dalvik VM [Bornstein 2008; Cheng and Buzbee 2010; Oh et al. 2012] on Android-based mobile devices.

Bebenita et al. [2010b] implemented trace-based compilation for the meta-circular Maxine JVM. The VM uses a nonoptimizing baseline JIT compiler instead of an interpreter, and was extended to generate instrumented code for trace recording. When an exception occurs during trace recording, they abort and invalidate the recorded traces. 
To avoid tail duplication, recorded traces are merged into trace regions with control flow merge points prior to JIT compilation. Bebenita's JIT compiler uses various loop optimizations and achieves excellent speedups for loop-intensive benchmarks, while performing worse than method-based compilation on benchmarks with fewer loops.

Based on the IBM J9/TR JVM, Inoue et al. [2011] implemented a trace-based JIT compiler. Their trace recording approach focuses on linear and cyclic traces without any join points except the head of cyclic traces. To reduce the transition overhead between interpreted and compiled code, a code sequence is generated for every potential trace exit to ensure that the stack is compatible to the interpreter. Such code sequences are also required for every bytecode that might throw an exception. They compare their trace-based JIT compiler to a slightly less optimizing version of their methodbased JIT compiler and achieve $96 \%$ of its peak performance on the DaCapo 9.12 Bach benchmarks. Wu et al. [2011] extended that work to avoid short-lived traces and unnecessary trace duplication. This reduces both the amount of generated machine code and the compilation time, while peak performance is not affected. When the application throws an exception during trace recording, the trace recording stops and the trace is stored. This is slightly better than aborting trace recording but still does not support that the exception source and exception handler are in the same compilation unit.

Our system targets Java and builds on an existing production-quality JVM. Similar to the work of Bebenita et al. [2010b], we detect loop headers using a static analysis on the Java bytecodes to avoid false loops. However, we do not solely focus on loops but allow traces to start at loop headers and method entries. Another major difference to other trace-based systems is our way of trace recording, which delays inlining decisions to the time of JIT compilation. At that point, more information is available than during trace recording so that sophisticated inlining heuristics (similar to those used in method-based compilers) can be used to control code bloat. Furthermore, we merge recorded traces into a trace graph before compilation [Häubl and Mössenböck 2011]. There, control flow merges are allowed, which addresses the problems with tail duplication so that complex traces can be handled efficiently. During compilation, we apply general and tracing-specific optimizations before generating machine code and we use the type information that is recorded in the traces to perform aggressive inlining of virtual calls. Unlike other trace-based compilers, we support the most efficient way of exception handling where the exception source and the exception handler are in the same compilation unit. Furthermore, we do not need to generate compensation code for every exception-throwing bytecode, as we rely on deoptimization instead.

\subsection{Method-Based Compilation}

Bradel and Abdelrahman [2005] recorded trace information to guide inlining decisions in a method-based compiler. An offline feedback-directed system was implemented for the Jikes RVM, which considers return instructions and backward branch targets as trace anchors. The system was evaluated on the benchmarks SPECjvm98 and Java Grande and resulted in a $10 \%$ higher performance while $47 \%$ more machine code was generated.

Partial-method compilation [Whaley 2001; Fink and Qian 2003] uses profiling information to detect infrequently executed method parts. Those parts are then excluded from compilation, so that fewer bytecodes are compiled. This reduces compilation time and increases startup performance. The Java HotSpot server compiler [Paleczny et al. $2001]$ is one example of a production-quality JIT compiler that uses this technique.

Suganuma et al. [2006] implemented region-based compilation where compilation heuristics and profiling data are used to exclude rarely executed method parts from compilation. Method inlining is used heavily to group frequently executed code into 
a single compilation unit, a so-called region. If a method part must be executed that was not compiled, the affected method is recompiled and on-stack-replaced so that the execution continues in the recompiled code. This approach reduces the compilation time by more than $20 \%$ and increases the performance by $5 \%$ on average for the benchmark suites SPECjvm98 and SPECjbb2000.

Our trace-based system also only compiles frequently executed method parts, and similar to a method-based compiler, it uses inlining to increase peak performance. However, it has the advantage that the recorded trace information is context sensitive and that we inline traces instead of methods. So, we can avoid inlining method parts that were executed frequently in total but that are not required for the current caller. Therefore, our trace-based compilation is more selective than partial-method compilation so that more aggressive inlining is possible without risking code bloat. We increase peak performance further by using the context-sensitive type information, which is recorded in the traces, for aggressive and optimistic optimizations. Another difference to methodbased JIT compilers is that we generalize on-stack replacement and establish loops as top-level compilation units so that they can be compiled and invoked independently from method traces. This increases the flexibility of the compiler when deciding about compilation unit boundaries. Our most efficient way of exception handling (where the exception source and its exception handler are within the same compilation unit) is similar to exception handling in a method-based compiler. However, the chance that the exception source and its exception handler end up in the same compilation unit is higher in our trace-based system because we inline more aggressively.

\section{CONCLUSIONS}

We presented a trace-based JIT compiler for Java that is integrated into Oracle's production-quality Java HotSpot VM. The runtime system supports traces that start at method entries or at loop headers, and we employ efficient calling conventions for invoking traces from interpreted and from compiled code. Furthermore, our trace-based compiler can handle exceptional control flow efficiently, which is an important factor to achieve good peak performance for complex Java applications.

By combining ideas from tracing and traditional method-based compilers, our system achieves a good peak performance while spending little time on JIT compilation. Compared to the Java HotSpot client compiler, we achieve up to $59 \%$ speedup (20\%, on average) for complex benchmarks such as DaCapo 9.12 Bach jython.

\section{ACKNOWLEDGMENTS}

This work has been supported by the Austrian Science Fund (FWF): project number P 22493-N18. Oracle and Java are registered trademarks of Oracle and/or its affiliates. Other names may be trademarks of their respective owners.

\section{REFERENCES}

Vasanth Bala, Evelyn Duesterwald, and Sanjeev Banerjia. 2000. Dynamo: A Transparent Dynamic Optimization System. In Proceedings of the ACM SIGPLAN Conference on Programming Language Design and Implementation. ACM, 1-12.

Michael Bebenita, Florian Brandner, Manuel Fahndrich, Francesco Logozzo, Wolfram Schulte, Nikolai Tillmann, and Herman Venter. 2010a. SPUR: A Trace-Based JIT Compiler for CIL. In Proceedings of the ACM International Conference on Object Oriented Programming Systems Languages and Applications. ACM, 708-725.

Michael Bebenita, Mason Chang, Gregor Wagner, Andreas Gal, Christian Wimmer, and Michael Franz. 2010b. Trace-Based Compilation in Execution Environments without Interpreters. In Proceedings of the International Conference on the Principles and Practice of Programming in Java. ACM, 59-68.

S. M. Blackburn, R. Garner, C. Hoffman, A. M. Khan, K. S. McKinley, R. Bentzur, A. Diwan, D. Feinberg, D. Frampton, S. Z. Guyer, M. Hirzel, A. Hosking, M. Jump, H. Lee, J. E. B. Moss, A. Phansalkar, D. 
Stefanović, T. VanDrunen, D. von Dincklage, and B. Wiedermann. 2006. The DaCapo benchmarks: Java benchmarking development and analysis. In Proceedings of the ACM SIGPLAN Conference on ObjectOriented Programming Systems, Languages, and Applications. ACM, 169-190.

Carl Friedrich Bolz, Antonio Cuni, Maciej Fijalkowski, and Armin Rigo. 2009. Tracing the Meta-Level: PyPy's Tracing JIT Compiler. In Proceedings of the Workshop on the Implementation, Compilation, Optimization of Object-Oriented Languages and Programming Systems. ACM Press, 18-25.

Dan Bornstein. 2008. Dalvik VM Internals. Presented at the Google I/O developer conference. Retrieved from http://sites.google.com/site/io/dalvik-vm-internals.

Borys J. Bradel and Tarek S. Abdelrahman. 2005. The Use of Traces for Inlining in Java Programs. In Proceedings of the International Conference on Languages and Compilers for High Performance Computing. Springer-Verlag, 179-193.

Mason Chang, Edwin Smith, Rick Reitmaier, Michael Bebenita, Andreas Gal, Christian Wimmer, Brendan Eich, and Michael Franz. 2009. Tracing for Web 3.0: Trace Compilation for the Next Generation Web Applications. In Proceedings of the ACM SIGPLAN/SIGOPS International Conference on Virtual Execution Environments. ACM, 71-80.

Ben Cheng and Bill Buzbee. 2010. A JIT Compiler for Android's Dalvik VM. Presented at the Google I/O developer conference. Retrieved from http://www.google.com/events/io/2010/sessions/jit-compilerandroids-dalvik-vm.html.

Ron Cytron, Jeanne Ferrante, Barry K. Rosen, Mark N. Wegman, and F. Kenneth Zadeck. 1991. Efficiently Computing Static Single Assignment Form and the Control Dependence Graph. ACM Transactions on Programming Languages and Systems 13, 4 (1991), 451-490.

Evelyn Duesterwald and Vasanth Bala. 2000. Software Profiling for Hot Path Prediction: Less is More. SIGPLAN Notices 35, 1 (2000), 202-211.

S. J. Fink and Feng Qian. 2003. Design, Implementation and Evaluation of Adaptive Recompilation with On-Stack Replacement. In Proceedings of the International Symposium on Code Generation and Optimization. IEEE Computer Society, 241-252.

Andreas Gal, Brendan Eich, Mike Shaver, David Anderson, David Mandelin, Mohammad R. Haghighat, Blake Kaplan, Graydon Hoare, Boris Zbarsky, Jason Orendorff, Jesse Ruderman, Edwin W. Smith, Rick Reitmaier, Michael Bebenita, Mason Chang, and Michael Franz. 2009. Trace-based Just-in-Time Type Specialization for Dynamic Languages. In Proceedings of the ACM SIGPLAN Conference on Programming Language Design and Implementation. ACM, 465-478.

Andreas Gal and Michael Franz. 2006. Incremental Dynamic Code Generation with Trace Trees. Technical Report. Donald Bren School of Information and Computer Science, University of California, Irvine.

Andreas Gal, Christian W. Probst, and Michael Franz. 2006. HotpathVM: An Effective JIT Compiler for Resource-constrained Devices. In Proceedings of the International Conference on Virtual Execution Environments. ACM, 144-153.

Robert Griesemer. 1999. Generation of Virtual Machine Code at Startup. In OOPSLA Workshop on Simplicity, Performance, and Portability in Virtual Machine Design. Sun Microsystems, Inc.

Christian Häubl and Hanspeter Mössenböck. 2011. Trace-based Compilation for the Java HotSpot Virtual Machine. In Proceedings of the International Conference on the Principles and Practice of Programming in Java. ACM, 129-138.

Christian Häubl, Christian Wimmer, and Hanspeter Mössenböck. 2012. Evaluation of Trace Inlining Heuristics for Java. In Proceedings of the ACM Symposium on Applied Computing. ACM, 1871-1876.

Christian Häubl, Christian Wimmer, and Hanspeter Mössenböck. 2013. Context-sensitive Trace Inlining for Java. Computer Languages, Systems and Structures 39, 4 (2013), 123-141.

Hiroshige Hayashizaki, Peng Wu, Hiroshi Inoue, Mauricio J. Serrano, and Toshio Nakatani. 2011. Improving the Performance of Trace-based Systems by False Loop Filtering. In Proceedings of the International Conference on Architectural Support for Programming Languages and Operating Systems. ACM Press, $405-418$.

David Hiniker, Kim Hazelwood, and Michael D. Smith. 2005. Improving Region Selection in Dynamic Optimization Systems. In Proceedings of the IEEE/ACM International Symposium on Microarchitecture. IEEE Computer Society, 141-154.

Urs Hölzle, Craig Chambers, and David Ungar. 1992. Debugging Optimized Code with Dynamic Deoptimization. In Proceedings of the ACM SIGPLAN Conference on Programming Language Design and Implementation. ACM, 32-43.

Urs Hölzle and David Ungar. 1994. Optimizing Dynamically-dispatched Calls with Run-time Type Feedback. In Proceedings of the ACM SIGPLAN Conference on Programming Language Design and Implementation. ACM, 326-336. 
Hiroshi Inoue, Hiroshige Hayashizaki, Peng Wu, and Toshio Nakatani. 2011. A Trace-based Java JIT Compiler Retrofitted from a Method-based Compiler. In Proceedings of the International Symposium on Code Generation and Optimization. IEEE Computer Society, 246-256.

Thomas Kotzmann, Christian Wimmer, Hanspeter Mössenböck, Thomas Rodriguez, Kenneth Russell, and David Cox. 2008. Design of the Java HotSpot Client Compiler for Java 6. ACM Transactions on Architecture and Code Optimization 5, 1, Article 7 (2008), 7:1-7:32 pages.

Hyeong-Seok Oh, Beom-Jun Kim, Hyung-Kyu Choi, and Soo-Mook Moon. 2012. Evaluation of Android Dalvik Virtual Machine. In Proceedings of the International Workshop on Java Technologies for Real-time and Embedded Systems. ACM, 115-124.

Oracle Corporation. 2013. Java Platform, Standard Edition 8 Developer Preview Releases. Retrieved from http://jdk8.java.net/download.html.

Michael Paleczny, Christopher Vick, and Cliff Click. 2001. The Java HotSpot Server Compiler. In Proceedings of the Java Virtual Machine Research and Technology Symposium. USENIX, 1-12.

Ian Rogers. 2002. Optimising Java Programs Through Basic Block Dynamic Compilation. Ph.D. Dissertation. Department of Computer Science, University of Manchester.

Standard Performance Evaluation Corporation. 2008. The SPECjum2008 Benchmarks. Retrieved from http:// www.spec.org/jvm2008/.

Toshio Suganuma, Toshiaki Yasue, and Toshio Nakatani. 2006. A Region-Based Compilation Technique for Dynamic Compilers. ACM Transactions on Programming Languages and Systems 28, 1 (2006), $134-174$.

John Whaley. 2001. Partial Method Compilation using Dynamic Profile Information. SIGPLAN Notices 36, 11 (2001), 166-179.

Christian Wimmer and Hanspeter Mössenböck. 2005. Optimized Interval Splitting in a Linear Scan Register Allocator. In Proceedings of the ACM / USENIX International Conference on Virtual Execution Environments. ACM, 132-141.

Peng Wu, Hiroshige Hayashizaki, Hiroshi Inoue, and Toshio Nakatani. 2011. Reducing Trace Selection Footprint for Large-scale Java Applications without Performance Loss. In Proceedings of the ACM International Conference on Object Oriented Programming Systems Languages and Applications. ACM, 789-804.

Received April 2013; revised November 2013; accepted January 2014 